\title{
NEUROIMMUNOLOGY
}

\section{Damage versus repair}

File name: NRNHL-

April-1

Word count: 330

Accompanying

picture:

File name of picture:

URLs
Complement, a component of the humoral immune system, is expressed by many cell types of the CNS. The role of complement in normal CNS function is unknown, but in disease or injury, such as ischaemia, complement activation contributes to the inflammatory response and, therefore, to resulting tissue destruction. However, new work from Marcela Pekna's laboratory identifies an additional and opposite role for complement in adult neurogenesis and ischaemia-induced repair.

Recent evidence from diverse sources has implicated the complement system in tissue regeneration. For example, in amphibians, C3 (third complement component) expression has been detected in regenerating limbs, and in newts both C3 and C5 (fifth component) have been detected in regenerating limbs and lenses. Furthermore, in mammals, C3 and C5 are both involved in hepatocyte proliferation and liver regeneration. These findings led Pekna's team to wonder if complement might have a role in adult neurogenesis.

Neurogenesis occurs as a normal function of the adult brain and becomes exaggerated in response to injury. First, the team investigated normal neurogenesis and found that mice deficient for $\mathrm{C} 3$, or its cell surface receptor $\mathrm{C} 3 \mathrm{AR}$, had a reduced number of newly formed and migrating neurons - indicating impaired neurogenesis. Next, to look at injury repair, the team artificially induced ischaemia and found that C3-deficient mice also had fewer new neurons at the repair site than wild-type mice. Importantly, these researchers showed that this reduction in ischaemia-induced neurogenesis was not due to a decreased injury size (as might occur as a result of reduced inflammation in the absence of C3).

It is surprising that complement can, on the one hand, be involved in inflammation and associated tissue damage, and, on the other hand, support neurogenesis and migration of new neurons. A better understanding of how these two opposing roles are balanced in the CNS might lead to therapies that can tip the scales in favour of repair.

Ruth Williams

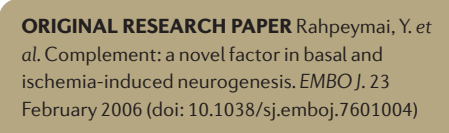

